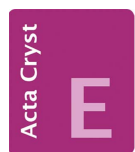

CRYSTALLOGRAPHIC COMMUNICATIONS

ISSN 2056-9890

Received 9 March 2016

Accepted 14 March 2016

Edited by $\mathrm{H}$. Stoeckli-Evans, University of Neuchâtel, Switzerland

Keywords: crystal structure; redetermination; hydrogen bonding; symmetric hydrogen bonds; refinement constraints; refinement restraints.

CCDC reference: 1465363

Supporting information: this article has supporting information at journals.iucr.org/e

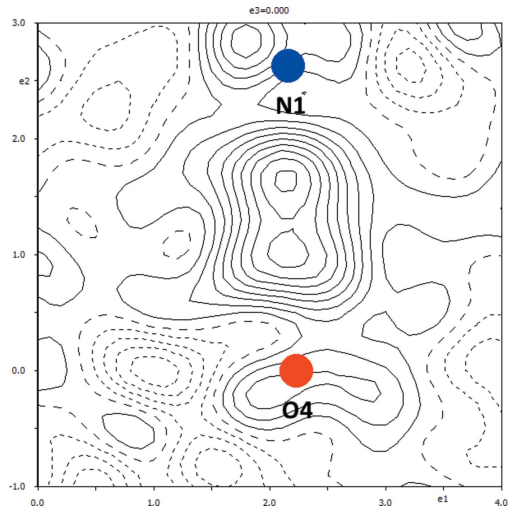

OPEN $\odot$ ACCESS

\section{A redetermination from the original data of the crystal structure of 2-amino-4,6-dimethoxy- pyrimidin-1-ium 4-aminobenzoate}

\author{
Jan Fábry*
}

Institute of Physics of the Czech Academy of Sciences, Na Slovance 2, 18221 Praha 8, Czech Republic. *Correspondence e-mail: fabry@fzu.cz

The title structure, $\mathrm{C}_{6} \mathrm{H}_{9.5} \mathrm{~N}_{3} \mathrm{O}_{2}{ }^{0.5+} \cdot \mathrm{C}_{7} \mathrm{H}_{6.5} \mathrm{NO}_{2}{ }^{0.5-}$, which might be named schematically as 2-amino-4,6-dimethoxypyrimidine-( $\mu_{2}$-hydrogen)-4-aminobenzoate to indicate a bridging $\mathrm{H}$ atom, has been redetermined from the data published by Thanigaimani, Muthiah \& Lynch [Acta Cryst. (2006), E62, o2976o2978]. The improvement of the present redetermination consists in a released geometry of the primary amine groups, which were originally assumed to be planar, as well as in a redetermination of the position of the hydroxy $\mathrm{H}$ atom. This $\mathrm{H}$ atom, whose parameters were originally constrained, turns out to be situated about the centre of the $\mathrm{O} \cdots \mathrm{N}$ hydrogen bond in two disordered positions with occupancies of 0.5 each.

\section{Chemical context}

Structures which contain hydroxyl, secondary and primary amine groups are often determined incorrectly because of an assumed geometry of these groups from which the applied constraints or restraints were inferred. In such cases, the correct geometry is missed as it is not verified by inspection of the difference electron-density maps. Thus, a considerable number of structures could have been determined more correctly - cf. Figs. 1 and 2 in Fábry et al. (2014). The inclusion of such structures causes bias in crystallographic databases such as the Cambridge Structural Database (CSD; Groom \& Allen, 2014).<smiles>COc1cc(OC)nc(N)n1</smiles>

In the course of recalculation of suspect structures which were retrieved from the CSD, defects in the structure determination of 2-amino-4,6-dimethoxypyrimidine-4-aminobenzoic acid (1/1) by Thanigaimani et al. (2006) have been found; the pertinent CSD refcode is IFACUO.

The defects in the original structure concern positional parameters both of the hydroxyl and the primary amine hydrogen atoms, which follow from unsubstantiated constraints of these hydrogen atoms. This means that the amine groups were assumed to be planar while the disorder regarding atom $\mathrm{H} 4$ was neglected because atom $\mathrm{H} 4$ was forced to be situated at atom $\mathrm{O} 4$. 


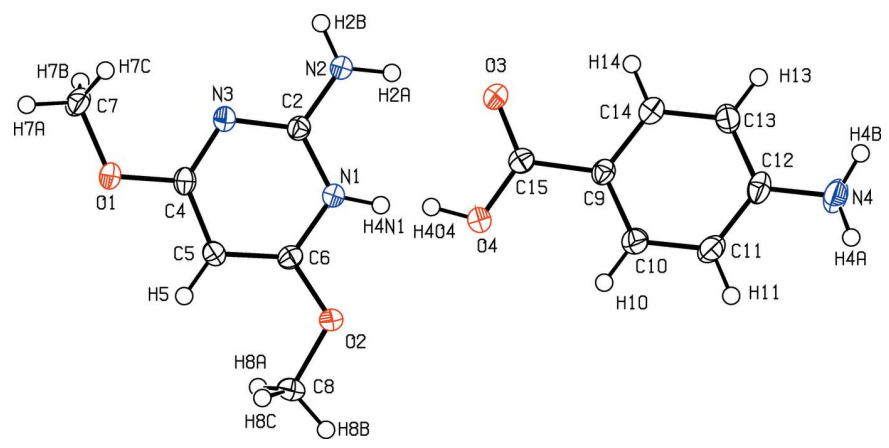

Figure 1

View of the constituent molecules of the title structure after the improved refinement. The displacement ellipsoids are depicted at the $50 \%$ probability level. The occupancies of atoms $\mathrm{H} 4 \mathrm{~N} 1$ and $\mathrm{H} 4 \mathrm{O} 4$ are each equal to 0.5 .

The aim of the present article is to demonstrate how the original structure determination can be improved.

\section{Structural commentary}

The structure of the title compound has been described by Thanigaimani et al. (2006) as 2-amino-4,6-dimethoxypyrimidine/4-aminobenzoic acid (1/1). In that article, the amine groups (centred on atoms N2 and N4) were assumed to be planar and were refined with distance constraints of $\mathrm{N}-\mathrm{H}$ $=0.86 \AA$. For the hydroxyl group $\mathrm{O} 4-\mathrm{H} 4$, atom $\mathrm{H} 4$ was refined with a distance constraint of $\mathrm{O} 4-\mathrm{H} 4=0.82 \AA$ $\left[U_{\text {iso }}\left(\mathrm{H}_{\text {primary amine }}\right)=1.2 U_{\text {eq }}\left(\mathrm{N}_{\text {primary amine }}\right)\right.$ while $U_{\text {iso }}(\mathrm{H} 4)=$ $\left.1.5 U_{\text {eq }}(\mathrm{O} 4)\right]$.

The improved refinement by JANA2006 (Petříček et al., 2014) yielded a non-planar geometry of the primary amine groups and a considerably different position for the hydrogen atom H4. This atom turns out to be disordered over two positions at about the centre of the hydrogen bond O4 $\cdots \mathrm{N} 1$ (Fig. 1). Thus, the title structure can be envisaged as an example of a structure with a symmetric hydrogen bond where the bridging hydrogen atom is disordered over two positions (see: for example Olovsson et al., 2002). One of these positions is closer to atom $\mathrm{N} 1$ while the other is closer to atom $\mathrm{O} 4$, and correspondingly they were labelled as $\mathrm{H} 4 n 1$ and $\mathrm{H} 4 o 4$. Each of the occupancies of $\mathrm{H} 4 n 1$ and $\mathrm{H} 4 o 4$ turned out to be equal to 0.5 within the interval given by the refined standard uncertainties; $c f$. the section of the electron density map in Fig. 2. The $\mathrm{pKa}$ of the conjugate acid to 2-amino-4,6-dimethoxypyrimidine is equal to 3.36 (Baldwin \& van den Broek, 1975), while $\mathrm{p} K \mathrm{a}_{1}$ and $\mathrm{p} K \mathrm{a}_{2}$ of 4-aminobenzoic acid are equal to 2.50 and 4.87, respectively ( $C R C$ Handbook of Chemistry and Physics, 2009). $\mathrm{pK} \mathrm{a}_{1}$ refers to the deprotonation of the hydrogen carboxylate into the carboxylate group, while $\mathrm{pKa}$ refers to the deprotonation of the ammonium group into the primary amine group in the solution ( $c f . \mathrm{pKa}$ for benzoic acid and aniline are equal to 4.20 and 4.87, respectively; $C R C$ Handbook of Chemistry and Physics, 2009). Thus, 2-amino4,6-dimethoxypyrimidine is a weaker acid while 4-aminobenzoic acid is a weaker base. These values favour the
Table 1

Hydrogen-bond geometry $\left(\AA,^{\circ}\right)$.

$\mathrm{Cg} 1$ is the centroid of the $\mathrm{C} 9-\mathrm{C} 14$ ring.

\begin{tabular}{lllll}
\hline$D-\mathrm{H} \cdots A$ & $D-\mathrm{H}$ & $\mathrm{H} \cdots A$ & $D \cdots A$ & $D-\mathrm{H} \cdots A$ \\
\hline $\mathrm{N} 1-\mathrm{H} 4 \mathrm{~N} 1 \cdots \mathrm{O} 4$ & $0.86(3)$ & $1.78(3)$ & $2.6459(14)$ & $176(2)$ \\
$\mathrm{O} 4-\mathrm{H} 4 \mathrm{O} 4 \cdots \mathrm{N} 1$ & $0.89(3)$ & $1.77(3)$ & $2.6459(14)$ & $170(3)$ \\
$\mathrm{N} 2-\mathrm{H} 2 \mathrm{~A} \cdots \mathrm{O} 3$ & $0.92(1)$ & $1.91(1)$ & $2.8163(14)$ & $172(1)$ \\
$\mathrm{N} 2-\mathrm{H} 2 \mathrm{~B} \cdots 3^{\mathrm{i}}$ & $0.88(1)$ & $2.04(1)$ & $2.8544(14)$ & $154(1)$ \\
$\mathrm{N} 4-\mathrm{H} 4 \mathrm{~A} \cdots \mathrm{O} 4^{\mathrm{ii}}$ & $0.92(2)$ & $2.27(2)$ & $3.1625(15)$ & $164(1)$ \\
$\mathrm{C} 7-\mathrm{H} 7 \mathrm{~B} \cdots \mathrm{O}^{\mathrm{iii}}$ & 0.96 & 2.59 & $3.4571(15)$ & 150 \\
$\mathrm{~N} 4-\mathrm{H} 4 b \cdots C g 1^{\text {iv }}$ & $0.89(2)$ & $2.724(15)$ & $3.5472(14)$ & $154.7(12)$ \\
\hline
\end{tabular}

Symmetry codes: (i) $-x+1,-y+2,-z$; (ii) $-x+1, y+\frac{1}{2},-z+\frac{1}{2}$; (iii) $-x,-y+1,-z$; (iv) $-x+2, y+\frac{1}{2},-z+\frac{1}{2}$.

formation of the salt rather than of the co-crystal. Since differences in the dissociation constants are relatively mild, the hydrogen atom is situated about the centre of the hydrogen bond $\mathrm{N} 1 \cdots \mathrm{O} 4$ and the structure in the solid state can be envisaged as a mixture of a co-crystal 2-amino-4,6dimethoxypyrimidine-4-aminobenzoic acid (1:1) with a salt 2amino-4,6-dimethoxypyrimidin-1-ium 4-aminobenzoate in a 1:1 proportion. Alternatively - as has been stated above - it can be assumed to be a structure with a disordered bridging hydrogen involved in a symmetric hydrogen bond (Olovsson et al., 2002).

In the recalculated structure determination, the deviation from planarity of the primary amine groups (including the $\mathrm{C}$ atoms to which they are attached) is larger for the one that is

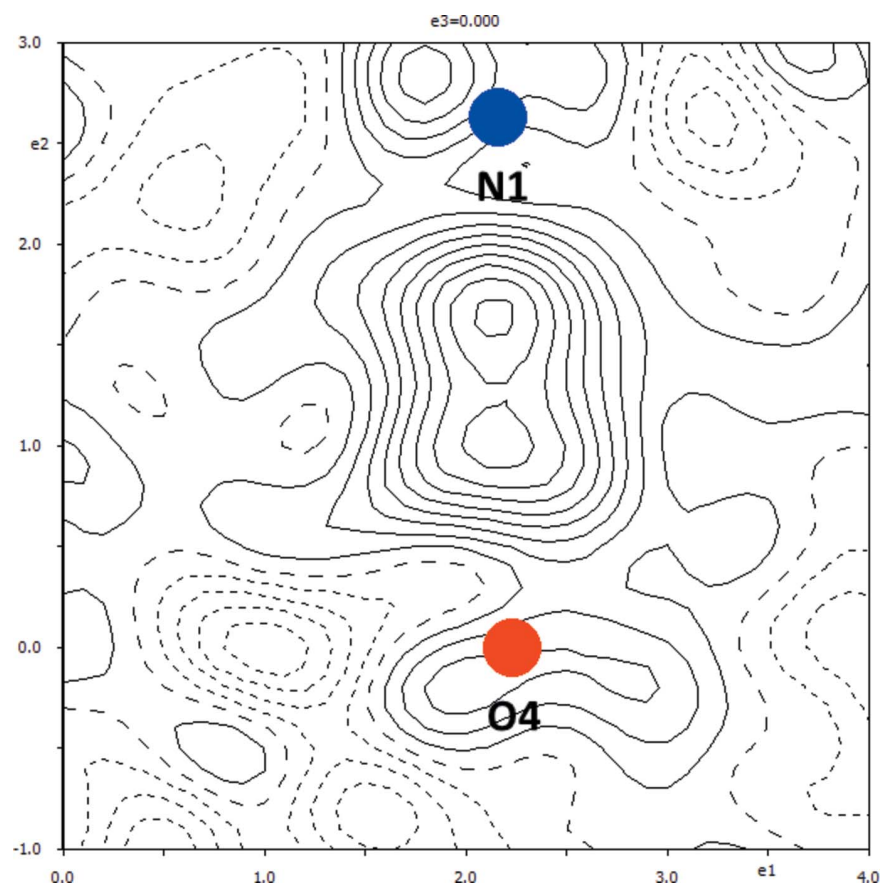

Figure 2

A section of the difference electron-density map for the present redetermined title structure, which shows the build-up of the electron density between the atom $\mathrm{O} 4$ (red) and N1 (blue). Positive and negative electron densities are indicated by continuous and dashed lines, respectively. The increment of electron density between neighbouring contours is 0.05 e $\AA^{-3}$ (JANA2006; Petříček et al., 2014). 


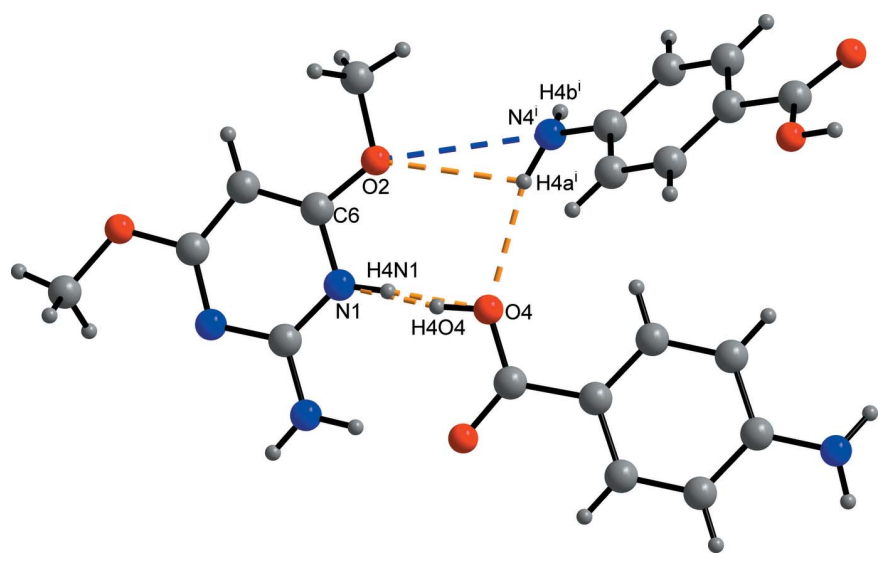

Figure 3

The section of the title structure which shows the graph-set motifs $R(7)_{2}^{3}$ $\mathrm{O} 4-\mathrm{H} 4 \mathrm{O} 4 \cdots \mathrm{N} 1-\mathrm{C} 6-\mathrm{O} 2 \cdots \mathrm{H} 4 a^{\mathrm{i}} \cdots \mathrm{N} 4^{\mathrm{i}}$ and $\mathrm{O} 4 \cdots \mathrm{H} 4 \mathrm{~N} 1-\mathrm{N} 1-\mathrm{C} 6-$ $\mathrm{O} 2 \cdots \mathrm{H} 4 a^{\mathrm{i}} \cdots \mathrm{N} 4^{\mathrm{i}}$ [symmetry code: (i) $-x+1, y-\frac{1}{2},-z+\frac{1}{2}$; colour code for atoms: grey $-\mathrm{C}$ and $\mathrm{H}$, blue $-\mathrm{N}$; red $-\mathrm{O}$; colour code for bonds: black: covalent bonds, dashed orange: $\mathrm{H} \cdots$ hydrogen-bond acceptor; blue $\mathrm{O} 2-$ N4: inclusion into the graph-set motif $\left.R(7)_{2}^{3}\right]$.

centred on N4 [C12-N4-H4a $115.4(9), \quad \mathrm{C} 12-\mathrm{N} 4-\mathrm{H} 4 a$ 114.5 (9), H4a-N4-H4b $\left.119.5(13)^{\circ}\right]$ than on N2 [C2-N2-H2a 119.5 (8), C2-N2-H2b 119.7 (8), H2a-N2-H2b 120.7 (12) $\left.{ }^{\circ}\right]$. This is in agreement with the longer bond length for $\mathrm{C} 12-\mathrm{N} 4$

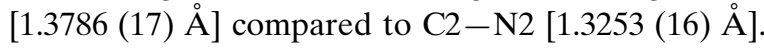

In a broader sense, the present redetermination emphasizes the importance of careful examination of the difference electron-density maps during structure determinations.

\section{Supramolecular features}

The details of the hydrogen bonding and the $\mathrm{N}-\mathrm{H} \cdots \pi$ electron ring interaction involving $\mathrm{N} 4-\mathrm{H} 4 b$ are given in Table 1. The graph-set motifs (Etter et al., 1990) were described by Thanigaimani et al. (2006) for the title structure. The graph-set motif $R_{2}^{3}(7)$ (Fig. 3) is shown in Fig. 2 of the article by Thanigaimani et al. (2006) and described there as $R_{2}^{2}(6)$.

In the present article, the graph-set motif $R_{2}^{3}(7)$ includes the atoms $\mathrm{O} 4-\mathrm{H} 404 \cdots \mathrm{N} 1-\mathrm{C} 6-\mathrm{O} 2 \cdots \mathrm{H} 4 a^{\mathrm{i}}-\mathrm{N} 4^{\mathrm{i}}$ or $\mathrm{O} 4 \cdots \mathrm{H} 4 n 1-\mathrm{N} 1-\mathrm{C} 6-\mathrm{O} 2 \cdots \mathrm{H} 4 a^{\mathrm{i}}-\mathrm{N} 4^{\mathrm{i}}$ on a local scale [Fig. 3; symmetry code (i): $-x+1, y-\frac{1}{2},-z+\frac{1}{2}$ ].

\section{Database survey}

The structure determination by Thanigaimani et al. (2006) has been included in the Cambridge Structural Database (Groom \& Allen, 2014) under the refcode IFACUO.

\section{Synthesis and crystallization}

The preparation of the title compound has been described by Thanigaimani et al. (2006).
Table 2

Experimental details.

\begin{tabular}{|c|c|}
\hline \multirow{2}{*}{\multicolumn{2}{|c|}{ Crystal data }} \\
\hline & \\
\hline Chemical formula & $\begin{array}{l}\mathrm{C}_{6} \mathrm{H}_{9.5} \mathrm{~N}_{3} \mathrm{O}_{2}{ }^{0.5+} \cdot \mathrm{C}_{7} \mathrm{H}_{6.5} \mathrm{NO}_{2}{ }^{0.5-} \\
2.30\end{array}$ \\
\hline $\begin{array}{l}M_{\mathrm{r}} \\
\text { Crystal system, space group }\end{array}$ & Monoclinic, $P 2_{1} / c$ \\
\hline Temperature $(\mathrm{K})$ & 293 \\
\hline$a, b, c(\AA)$ & $6.6358(4), 7.5560(5), 27.4226(16)$ \\
\hline$\beta\left({ }^{\circ}\right)$ & $94.418(2)$ \\
\hline$V\left(\mathrm{~A}^{3}\right)$ & $1370.89(15)$ \\
\hline$Z$ & 4 \\
\hline Radiation type & Мо $K \alpha$ \\
\hline$\mu\left(\mathrm{mm}^{-1}\right)$ & 0.11 \\
\hline Crystal size $(\mathrm{mm})$ & $0.44 \times 0.32 \times 0.08$ \\
\hline \multicolumn{2}{|l|}{ Data collection } \\
\hline Diffractometer & $\begin{array}{l}\text { Bruker-Nonius KappaCCD area- } \\
\text { detector }\end{array}$ \\
\hline $\begin{array}{l}\text { No. of measured, independent and } \\
\text { observed }[I>3 \sigma(I)] \text { reflections }\end{array}$ & $14577,3130,2302$ \\
\hline$R_{\text {int }}$ & 0.032 \\
\hline$(\sin \theta / \lambda)_{\max }\left(\AA^{-1}\right)$ & 0.651 \\
\hline \multicolumn{2}{|l|}{ Refinement } \\
\hline$R\left[F^{2}>3 \sigma\left(F^{2}\right)\right], w R\left(F^{2}\right), S$ & $0.037,0.084,1.91$ \\
\hline No. of reflections & 3130 \\
\hline No. of parameters & 208 \\
\hline $\mathrm{H}$-atom treatment & $\begin{array}{l}\mathrm{H} \text { atoms treated by a mixture of } \\
\text { independent and constrained } \\
\text { refinement }\end{array}$ \\
\hline$\Delta \rho_{\max }, \Delta \rho_{\min }\left(\mathrm{e} \AA^{-3}\right)$ & $0.21,-0.23$ \\
\hline
\end{tabular}

Computer programs: DENZO (Otwinowski \& Minor, 1997), COLLECT (Hooft, 1998), SHELXS97 (Sheldrick, 2008), PLATON (Spek, 2009), DIAMOND (Brandenburg \& Putz, 2005) and JANA2006 (Petříček et al., 2014).

\section{Refinement}

Crystal data, data collection and structure refinement details are summarized in Table 2. All the hydrogen atoms were discernible in the difference electron-density map. The aryl hydrogen atoms were constrained by the constraints $\mathrm{C}_{\text {aryl }}-$ $\mathrm{H}_{\text {aryl }}=0.93 \AA$ and $U_{\text {iso }}\left(\mathrm{H}_{\text {aryl }}\right)=1.2 U_{\text {eq }}\left(\mathrm{C}_{\text {aryl }}\right)$ while the methyl hydrogens were constrained by the constraints $\mathrm{C}_{\text {methyl }}-$ $\mathrm{H}_{\text {methyl }}=0.96 \AA$ and $U_{\text {iso }}\left(\mathrm{H}_{\text {methyl }}\right)=1.5 U_{\text {eq }}\left(\mathrm{C}_{\text {methyl }}\right)$. The hydrogen atoms of the primary amine group $\mathrm{N} 2$ were constrained by $U_{\text {iso }}\left(\mathrm{H}_{\mathrm{N} 2}\right)=1.2 U_{\mathrm{eq}}(\mathrm{N} 2)$. The displacement parameters of the hydroxyl hydrogen $\mathrm{H} 4 \mathrm{O} 4$ and of the secondary amine $\mathrm{H} 4 \mathrm{~N} 1$ were constrained by $U_{\text {iso }}(\mathrm{H} 4 \mathrm{O} 4)=$ $1.5 U_{\text {eq }}(\mathrm{O} 4)$ and $U_{\text {iso }}(\mathrm{H} 4 \mathrm{~N} 1)=1.5 U_{\text {eq }}(\mathrm{N} 1)$ while their positional parameters were refined freely.

The model with the refinement of the occupational factors of $\mathrm{H} 4 \mathrm{~N} 1$ and $\mathrm{H} 4 \mathrm{O} 4$ under the condition that the sum of these occupational factors should equal to 1 resulted in the values 0.499 (25) and 0.501 (25), respectively. Therefore the occupational parameters were set to 0.5 in the final model and not further refined.

\section{Acknowledgements}

The author expresses gratitude for the support provided by Project NPU I - LO1603 of the Ministry of Education of the Czech Republic. Discussions with Dr Radek Cibulka and Igor 
Linhart from the Chemical University in Prague are gratefully acknowledged.

\section{References}

Baldwin, D. \& van den Broek, P. (1975). J. Chem. Soc. Perkin Trans. 1, pp. 375-377.

Brandenburg, K. \& Putz, H. (2005). DIAMOND. Crystal Impact GbR, Bonn, Germany.

CRC Handbook of Chemistry and Physics (2009). Editor-in-chief D. R. Lidl, 90th ed. Boca Raton: CRC Press.

Etter, M. C., MacDonald, J. C. \& Bernstein, J. (1990). Acta Cryst. B46, 256-262.

Fábry, J., Dušek, M., Vaněk, P., Rafalovskyi, I., Hlinka, J. \& Urban, J. (2014). Acta Cryst. C70, 1153-1160.
Groom, C. R. \& Allen, F. H. (2014). Angew. Chem. Int. Ed. 53, 662671.

Hooft, R. (1998). COLLECT. Nonius BV, Delft, The Netherlands.

Olovsson, I., Ptasiewicz-Bak, H., Gustafsson, T. \& Majerz, I. (2002). Acta Cryst. B58, 627-631.

Otwinowski, Z. \& Minor, W. (1997). Methods in Enzymology, Vol. 276, Macromolecular Crystallography, part A, edited by C. W. Carter Jr. \& R. M. Sweet, pp. 307-326. New York: Academic Press.

Petrríček, V., Dušek, M. \& Palatinus, L. (2014). Z. Kristallogr. 229, 345-352.

Sheldrick, G. M. (2008). Acta Cryst. A64, 112-122.

Spek, A. L. (2009). Acta Cryst. D65, 148-155.

Thanigaimani, K., Muthiah, P. T. \& Lynch, D. E. (2006). Acta Cryst. E62, o2976-o2978. 


\section{supporting information}

Acta Cryst. (2016). E72, 512-515 [https://doi.org/10.1107/S2056989016004321]

\section{A redetermination from the original data of the crystal structure of 2- amino-4,6-dimethoxypyrimidin-1-ium 4-aminobenzoate}

\section{Jan Fábry}

Computing details

Data collection: DENZO (Otwinowski \& Minor, 1997) and COLLECT (Hooft, 1998); cell refinement: DENZO

(Otwinowski \& Minor, 1997) and COLLECT (Hooft, 1998); data reduction: DENZO (Otwinowski \& Minor, 1997) and COLLECT (Hooft, 1998); program(s) used to solve structure: SHELXS97 (Sheldrick, 2008); program(s) used to refine structure: JANA2006 (Petříček et al., 2014); molecular graphics: PLATON (Spek, 2009), DIAMOND (Brandenburg \& Putz, 2005) and JANA2006 (Petříček et al., 2014); software used to prepare material for publication: JANA2006 (Petříček et al., 2014).

2-Amino-4,6-dimethoxypyrimidin-1-ium 4-aminobenzoate

\section{Crystal data}

$\mathrm{C}_{6} \mathrm{H}_{9.5} \mathrm{~N}_{3} \mathrm{O}_{2} 0.5+\cdot \mathrm{C}_{7} \mathrm{H}_{6.5} \mathrm{NO}_{2} 0.5--$

$M_{r}=292.30$

Monoclinic, $P 2{ }_{1} / c$

Hall symbol: -P $2 \mathrm{ybc}$

$a=6.6358(4) \AA$

$b=7.5560(5) \AA$

$c=27.4226(16) \AA$

$\beta=94.418(2)^{\circ}$

$V=1370.89(15) \AA^{3}$

$Z=4$

\section{Data collection}

Bruker-Nonius KappaCCD area-detector diffractometer

Radiation source: Bruker-Nonius FR591 rotating anode

Graphite monochromator

$\varphi$ and $\omega$ scans

14577 measured reflections

Refinement

Refinement on $F^{2}$

$R[F>3 \sigma(F)]=0.037$

$w R(F)=0.084$

$S=1.91$

3130 reflections

208 parameters
$F(000)=616$

$D_{\mathrm{x}}=1.416 \mathrm{Mg} \mathrm{m}^{-3}$

Mo $K \alpha$ radiation, $\lambda=0.71073 \AA$

Cell parameters from 50 reflections

$\theta=3.5-27.5^{\circ}$

$\mu=0.11 \mathrm{~mm}^{-1}$

$T=293 \mathrm{~K}$

Block, colourless

$0.44 \times 0.32 \times 0.08 \mathrm{~mm}$

3130 independent reflections

2302 reflections with $I>3 \sigma(I)$

$R_{\text {int }}=0.032$

$\theta_{\max }=27.5^{\circ}, \theta_{\min }=3.5^{\circ}$

$h=-8 \rightarrow 8$

$k=-9 \rightarrow 9$

$l=-35 \rightarrow 35$

0 restraints

50 constraints

$\mathrm{H}$ atoms treated by a mixture of independent and constrained refinement

Weighting scheme based on measured s.u.'s $w=$ $1 /\left(\sigma^{2}(I)+0.0004 I^{2}\right)$ 
$(\Delta / \sigma)_{\max }=0.021$

$\Delta \rho_{\max }=0.21$ e $\AA^{-3}$

$$
\Delta \rho_{\min }=-0.23 \text { e } \AA^{-3}
$$

Special details

Refinement. This part differs from the original article by Thanigaimani et al. (2006). It also differs from the refinement by Thanigaimani et al. (2006) by a different threshold for the consideration of the observed diffractions: $\mathrm{F}^{2}>3 \sigma\left(\mathrm{F}^{2}\right)$ has been used as criterion for observed diffractions by JANA2006 which was used for the calculation of the corrected structural model.

Fractional atomic coordinates and isotropic or equivalent isotropic displacement parameters $\left(\AA^{2}\right)$

\begin{tabular}{|c|c|c|c|c|c|}
\hline & $x$ & $y$ & $z$ & $U_{\text {iso }} * / U_{\text {eq }}$ & Occ. $(<1)$ \\
\hline $\mathrm{O} 1$ & $-0.31928(12)$ & $0.65273(11)$ & $-0.06555(3)$ & $0.0245(3)$ & \\
\hline $\mathrm{O} 2$ & $-0.10295(12)$ & $0.62305(10)$ & $0.10293(3)$ & $0.0220(3)$ & \\
\hline N1 & $0.08195(15)$ & $0.75282(13)$ & $0.04894(4)$ & $0.0190(3)$ & \\
\hline N2 & $0.29136(17)$ & $0.87608(15)$ & $-0.00426(4)$ & $0.0257(4)$ & \\
\hline N3 & $-0.01303(15)$ & $0.76827(13)$ & -0.03671 & $0.0195(3)$ & \\
\hline $\mathrm{C} 2$ & $0.11662(18)$ & $0.79867(15)$ & $0.00265(4)$ & $0.0187(4)$ & \\
\hline $\mathrm{C} 4$ & $-0.18261(18)$ & $0.68662(15)$ & $-0.02786(4)$ & $0.0194(4)$ & \\
\hline $\mathrm{C} 5$ & $-0.23220(18)$ & $0.62937(15)$ & $0.01815(4)$ & $0.0201(4)$ & \\
\hline C6 & $-0.09140(18)$ & $0.66647(15)$ & $0.05581(4)$ & $0.0186(4)$ & \\
\hline $\mathrm{C} 7$ & $-0.26609(18)$ & $0.70054(18)$ & $-0.11374(4)$ & $0.0261(4)$ & \\
\hline $\mathrm{C} 8$ & $-0.26910(18)$ & $0.51184(16)$ & $0.11446(4)$ & $0.0244(4)$ & \\
\hline $\mathrm{O} 3$ & $0.54244(13)$ & $0.93439(11)$ & $0.08178(3)$ & $0.0263(3)$ & \\
\hline $\mathrm{O} 4$ & $0.31630(13)$ & $0.82158(12)$ & $0.12916(3)$ & $0.0258(3)$ & \\
\hline N4 & $0.9485(2)$ & $1.16699(16)$ & $0.28973(4)$ & $0.0366(4)$ & \\
\hline $\mathrm{C} 9$ & $0.60450(18)$ & $0.96745(15)$ & $0.16726(4)$ & $0.0198(4)$ & \\
\hline $\mathrm{C} 10$ & $0.5334(2)$ & $0.95948(16)$ & $0.21374(5)$ & $0.0252(4)$ & \\
\hline $\mathrm{C} 11$ & $0.6456(2)$ & $1.02612(16)$ & $0.25407(5)$ & $0.0286(4)$ & \\
\hline $\mathrm{C} 12$ & $0.8340(2)$ & $1.10412(16)$ & $0.24933(4)$ & 0.0251 (4) & \\
\hline $\mathrm{C} 13$ & $0.90630(19)$ & $1.11079(16)$ & $0.20281(5)$ & $0.0254(4)$ & \\
\hline $\mathrm{C} 14$ & $0.79433(19)$ & $1.04458(15)$ & $0.16262(5)$ & $0.0227(4)$ & \\
\hline $\mathrm{C} 15$ & $0.48369(18)$ & $0.90491(15)$ & $0.12288(4)$ & $0.0202(4)$ & \\
\hline $\mathrm{H} 2 \mathrm{a}$ & $0.3827(19)$ & 0.8954 (17) & $0.0219(5)$ & $0.0308^{*}$ & \\
\hline $\mathrm{H} 2 \mathrm{~b}$ & $0.3152(19)$ & $0.9139(17)$ & $-0.0336(5)$ & $0.0308^{*}$ & \\
\hline H5 & -0.352188 & 0.570355 & 0.022827 & $0.0241^{*}$ & \\
\hline $\mathrm{H} 7 \mathrm{a}$ & -0.38336 & 0.691873 & -0.13641 & $0.0391 *$ & \\
\hline $\mathrm{H} 7 \mathrm{~b}$ & -0.163259 & 0.621794 & -0.123627 & $0.0391 *$ & \\
\hline $\mathrm{H} 7 \mathrm{c}$ & -0.216204 & 0.819832 & -0.113329 & $0.0391 *$ & \\
\hline $\mathrm{H} 8 \mathrm{a}$ & -0.266284 & 0.403993 & 0.096019 & $0.0365^{*}$ & \\
\hline $\mathrm{H} 8 \mathrm{~b}$ & -0.257451 & 0.484961 & 0.148775 & $0.0365^{*}$ & \\
\hline $\mathrm{H} 8 \mathrm{c}$ & -0.394276 & 0.572471 & 0.106262 & $0.0365^{*}$ & \\
\hline $\mathrm{H} 4 \mathrm{a}$ & $0.878(2)$ & $1.1906(19)$ & $0.3166(6)$ & $0.0439 *$ & \\
\hline $\mathrm{H} 4 \mathrm{~b}$ & $1.050(2)$ & $1.237(2)$ & $0.2829(5)$ & $0.0439 *$ & \\
\hline H10 & 0.408167 & 0.908373 & 0.217631 & $0.0302 *$ & \\
\hline H11 & 0.595299 & 1.019027 & 0.284732 & $0.0344 *$ & \\
\hline H13 & 1.032145 & 1.160827 & 0.198977 & $0.0305^{*}$ & \\
\hline H14 & 0.845086 & 1.051013 & 0.131987 & $0.0273 *$ & \\
\hline H4N1 & $0.157(5)$ & $0.771(4)$ & $0.0756(12)$ & $0.0285^{*}$ & 0.5 \\
\hline
\end{tabular}


0.5

Atomic displacement parameters $\left(\AA^{2}\right)$

\begin{tabular}{lllllll}
\hline & $U^{11}$ & $U^{22}$ & $U^{33}$ & $U^{12}$ & $U^{13}$ & $U^{23}$ \\
\hline O1 & $0.0216(5)$ & $0.0332(5)$ & $0.0179(5)$ & $-0.0044(4)$ & $-0.0040(4)$ & $0.0017(4)$ \\
O2 & $0.0211(5)$ & $0.0274(5)$ & $0.0173(5)$ & $-0.0059(4)$ & $0.0009(4)$ & $0.0032(4)$ \\
N1 & $0.0188(6)$ & $0.0218(6)$ & $0.0161(5)$ & $-0.0025(5)$ & $0.0004(4)$ & $-0.0001(4)$ \\
N2 & $0.0232(6)$ & $0.0371(7)$ & $0.0165(6)$ & $-0.0107(5)$ & $-0.0004(5)$ & $0.0015(5)$ \\
N3 & $0.0190(6)$ & $0.0212(5)$ & $0.0179(5)$ & $-0.0008(4)$ & $-0.0012(4)$ & $-0.0006(4)$ \\
C2 & $0.0203(7)$ & $0.0184(6)$ & $0.0173(6)$ & $-0.0003(5)$ & $0.0008(5)$ & $-0.0010(5)$ \\
C4 & $0.0188(7)$ & $0.0188(6)$ & $0.0201(6)$ & $0.0027(5)$ & $-0.0026(5)$ & $-0.0015(5)$ \\
C5 & $0.0178(7)$ & $0.0212(6)$ & $0.0211(7)$ & $-0.0029(5)$ & $0.0000(5)$ & $0.0002(5)$ \\
C6 & $0.0212(7)$ & $0.0171(6)$ & $0.0175(6)$ & $0.0015(5)$ & $0.0024(5)$ & $0.0007(5)$ \\
C7 & $0.0260(7)$ & $0.0347(8)$ & $0.0169(7)$ & $0.0014(6)$ & $-0.0024(6)$ & $0.0022(5)$ \\
C8 & $0.0228(7)$ & $0.0268(7)$ & $0.0239(7)$ & $-0.0055(6)$ & $0.0043(6)$ & $0.0043(5)$ \\
O3 & $0.0258(5)$ & $0.0358(5)$ & $0.0172(5)$ & $-0.0057(4)$ & $0.0016(4)$ & $-0.0019(4)$ \\
O4 & $0.0243(5)$ & $0.0324(5)$ & $0.0204(5)$ & $-0.0089(4)$ & $-0.0002(4)$ & $0.0003(4)$ \\
N4 & $0.0480(8)$ & $0.0393(7)$ & $0.0209(6)$ & $-0.0112(6)$ & $-0.0072(6)$ & $-0.0028(5)$ \\
C9 & $0.0236(7)$ & $0.0177(6)$ & $0.0176(6)$ & $0.0004(5)$ & $-0.0009(5)$ & $0.0006(5)$ \\
C10 & $0.0294(8)$ & $0.0246(7)$ & $0.0214(7)$ & $-0.0043(6)$ & $0.0017(6)$ & $0.0008(5)$ \\
C11 & $0.0400(9)$ & $0.0287(7)$ & $0.0171(7)$ & $-0.0048(6)$ & $0.0017(6)$ & $0.0005(5)$ \\
C12 & $0.0341(8)$ & $0.0189(6)$ & $0.0207(7)$ & $0.0012(6)$ & $-0.0076(6)$ & $-0.0002(5)$ \\
C13 & $0.0239(7)$ & $0.0261(7)$ & $0.0255(7)$ & $-0.0033(6)$ & $-0.0031(6)$ & $0.0001(5)$ \\
C14 & $0.0252(7)$ & $0.0240(7)$ & $0.0189(7)$ & $0.0016(6)$ & $0.0004(6)$ & $0.0002(5)$ \\
C15 & $0.0211(7)$ & $0.0196(6)$ & $0.0199(7)$ & $0.0019(5)$ & $0.0017(5)$ & $0.0010(5)$ \\
& & & & & & \\
\hline
\end{tabular}

Geometric parameters $\left(\AA,{ }^{\circ}\right)$

\begin{tabular}{llll}
\hline $\mathrm{O} 1-\mathrm{C} 4$ & $1.3462(14)$ & $\mathrm{O} 4-\mathrm{H} 4 \mathrm{O} 4$ & $0.89(3)$ \\
$\mathrm{O} 1-\mathrm{C} 7$ & $1.4400(14)$ & $\mathrm{N} 4-\mathrm{C} 12$ & $1.3786(17)$ \\
$\mathrm{O} 2-\mathrm{C} 6$ & $1.3411(14)$ & $\mathrm{N} 4-\mathrm{H} 4 \mathrm{a}$ & $0.918(15)$ \\
$\mathrm{O} 2-\mathrm{C} 8$ & $1.4405(15)$ & $\mathrm{N} 4-\mathrm{H} 4 \mathrm{~b}$ & $0.886(15)$ \\
$\mathrm{N} 1-\mathrm{C} 2$ & $1.3525(16)$ & $\mathrm{C} 9-\mathrm{C} 10$ & $1.3940(17)$ \\
$\mathrm{N} 1-\mathrm{C} 6$ & $1.3483(16)$ & $\mathrm{C} 9-\mathrm{C} 14$ & $1.4026(17)$ \\
$\mathrm{N} 1-\mathrm{H} 4 \mathrm{~N} 1$ & $0.86(3)$ & $\mathrm{C} 9-\mathrm{C} 15$ & $1.4821(16)$ \\
$\mathrm{N} 2-\mathrm{C} 2$ & $1.3253(16)$ & $\mathrm{C} 10-\mathrm{C} 11$ & $1.3799(17)$ \\
$\mathrm{N} 2-\mathrm{H} 2 \mathrm{a}$ & $0.914(13)$ & $\mathrm{C} 10-\mathrm{H} 10$ & 0.93 \\
$\mathrm{~N} 2-\mathrm{H} 2 \mathrm{~b}$ & $0.880(14)$ & $\mathrm{C} 11-\mathrm{C} 12$ & $1.3973(19)$ \\
$\mathrm{N} 3-\mathrm{C} 2$ & $1.3472(15)$ & $\mathrm{C} 11-\mathrm{H} 11$ & 0.93 \\
$\mathrm{~N} 3-\mathrm{C} 4$ & $1.3221(15)$ & $\mathrm{C} 12-\mathrm{C} 13$ & $1.3980(18)$ \\
$\mathrm{C} 4-\mathrm{C} 5$ & $1.3969(17)$ & $\mathrm{C} 13-\mathrm{C} 14$ & $1.3751(17)$ \\
$\mathrm{C} 5-\mathrm{C} 6$ & $1.3672(16)$ & $\mathrm{C} 13-\mathrm{H} 13$ & 0.93 \\
$\mathrm{C} 5-\mathrm{H} 5$ & 0.93 & $\mathrm{C} 14-\mathrm{H} 14$ & 0.93 \\
$\mathrm{C} 7-\mathrm{H} 7 \mathrm{a}$ & 0.96 & $\mathrm{H} 2 \mathrm{a}-\mathrm{H} 2 \mathrm{~b}$ & $1.559(19)$ \\
$\mathrm{C} 7-\mathrm{H} 7 \mathrm{~b}$ & 0.96 & $\mathrm{H} 7 \mathrm{a}-\mathrm{H} 7 \mathrm{~b}$ & 1.5677 \\
$\mathrm{C} 7-\mathrm{H} 7 \mathrm{c}$ & 0.96 & $\mathrm{H} 7 \mathrm{a}-\mathrm{H} 7 \mathrm{c}$ & 1.5677 \\
$\mathrm{C} 8-\mathrm{H} 8 \mathrm{a}$ & 0.96 & $\mathrm{H} 7 \mathrm{~b}-\mathrm{H} 7 \mathrm{c}$ &
\end{tabular}




\begin{tabular}{|c|c|c|c|}
\hline $\mathrm{C} 8-\mathrm{H} 8 \mathrm{~b}$ & 0.96 & $\mathrm{H} 8 \mathrm{a}-\mathrm{H} 8 \mathrm{~b}$ & 1.5677 \\
\hline $\mathrm{C} 8-\mathrm{H} 8 \mathrm{c}$ & 0.96 & $\mathrm{H} 8 \mathrm{a}-\mathrm{H} 8 \mathrm{c}$ & 1.5677 \\
\hline $\mathrm{O} 3-\mathrm{C} 15$ & $1.2409(15)$ & $\mathrm{H} 8 \mathrm{~b}-\mathrm{H} 8 \mathrm{c}$ & 1.5677 \\
\hline $\mathrm{O} 4-\mathrm{C} 15$ & $1.2998(15)$ & $\mathrm{H} 4 \mathrm{a}-\mathrm{H} 4 \mathrm{~b}$ & $1.56(2)$ \\
\hline $\mathrm{C} 4-\mathrm{O} 1-\mathrm{C} 7$ & $117.31(9)$ & $\mathrm{H} 8 \mathrm{a}-\mathrm{C} 8-\mathrm{H} 8 \mathrm{~b}$ & 109.47 \\
\hline $\mathrm{C} 6-\mathrm{O} 2-\mathrm{C} 8$ & $117.11(8)$ & $\mathrm{H} 8 \mathrm{a}-\mathrm{C} 8-\mathrm{H} 8 \mathrm{c}$ & 109.47 \\
\hline $\mathrm{C} 2-\mathrm{N} 1-\mathrm{C} 6$ & $117.63(10)$ & $\mathrm{H} 8 \mathrm{~b}-\mathrm{C} 8-\mathrm{H} 8 \mathrm{c}$ & 109.47 \\
\hline $\mathrm{C} 2-\mathrm{N} 1-\mathrm{H} 4 \mathrm{~N} 1$ & $129(2)$ & $\mathrm{C} 15-\mathrm{O} 4-\mathrm{H} 4 \mathrm{O} 4$ & $110(2)$ \\
\hline $\mathrm{C} 6-\mathrm{N} 1-\mathrm{H} 4 \mathrm{~N} 1$ & $114(2)$ & $\mathrm{C} 12-\mathrm{N} 4-\mathrm{H} 4 \mathrm{a}$ & $115.4(9)$ \\
\hline $\mathrm{C} 2-\mathrm{N} 2-\mathrm{H} 2 \mathrm{a}$ & $119.5(8)$ & $\mathrm{C} 12-\mathrm{N} 4-\mathrm{H} 4 \mathrm{~b}$ & $114.5(9)$ \\
\hline $\mathrm{C} 2-\mathrm{N} 2-\mathrm{H} 2 \mathrm{~b}$ & $119.7(8)$ & $\mathrm{H} 4 \mathrm{a}-\mathrm{N} 4-\mathrm{H} 4 \mathrm{~b}$ & $119.5(13)$ \\
\hline $\mathrm{H} 2 \mathrm{a}-\mathrm{N} 2-\mathrm{H} 2 \mathrm{~b}$ & $120.7(12)$ & $\mathrm{C} 10-\mathrm{C} 9-\mathrm{C} 14$ & $118.07(11)$ \\
\hline $\mathrm{C} 2-\mathrm{N} 3-\mathrm{C} 4$ & $115.62(10)$ & $\mathrm{C} 10-\mathrm{C} 9-\mathrm{C} 15$ & $122.49(11)$ \\
\hline $\mathrm{N} 1-\mathrm{C} 2-\mathrm{N} 2$ & $117.46(11)$ & $\mathrm{C} 14-\mathrm{C} 9-\mathrm{C} 15$ & $119.39(11)$ \\
\hline $\mathrm{N} 1-\mathrm{C} 2-\mathrm{N} 3$ & $124.33(11)$ & $\mathrm{C} 9-\mathrm{C} 10-\mathrm{C} 11$ & $121.15(12)$ \\
\hline $\mathrm{N} 2-\mathrm{C} 2-\mathrm{N} 3$ & $118.20(11)$ & $\mathrm{C} 9-\mathrm{C} 10-\mathrm{H} 10$ & 119.43 \\
\hline $\mathrm{O} 1-\mathrm{C} 4-\mathrm{N} 3$ & $118.71(10)$ & $\mathrm{C} 11-\mathrm{C} 10-\mathrm{H} 10$ & 119.43 \\
\hline $\mathrm{O} 1-\mathrm{C} 4-\mathrm{C} 5$ & $116.29(10)$ & $\mathrm{C} 10-\mathrm{C} 11-\mathrm{C} 12$ & $120.73(12)$ \\
\hline $\mathrm{N} 3-\mathrm{C} 4-\mathrm{C} 5$ & $125.00(10)$ & $\mathrm{C} 10-\mathrm{C} 11-\mathrm{H} 11$ & 119.64 \\
\hline $\mathrm{C} 4-\mathrm{C} 5-\mathrm{C} 6$ & $115.08(11)$ & $\mathrm{C} 12-\mathrm{C} 11-\mathrm{H} 11$ & 119.64 \\
\hline $\mathrm{C} 4-\mathrm{C} 5-\mathrm{H} 5$ & 122.46 & $\mathrm{~N} 4-\mathrm{C} 12-\mathrm{C} 11$ & $120.82(12)$ \\
\hline $\mathrm{C} 6-\mathrm{C} 5-\mathrm{H} 5$ & 122.46 & $\mathrm{~N} 4-\mathrm{C} 12-\mathrm{C} 13$ & $120.96(12)$ \\
\hline $\mathrm{O} 2-\mathrm{C} 6-\mathrm{N} 1$ & $111.45(10)$ & $\mathrm{C} 11-\mathrm{C} 12-\mathrm{C} 13$ & $118.18(11)$ \\
\hline $\mathrm{O} 2-\mathrm{C} 6-\mathrm{C} 5$ & $126.24(11)$ & $\mathrm{C} 12-\mathrm{C} 13-\mathrm{C} 14$ & $121.08(12)$ \\
\hline $\mathrm{N} 1-\mathrm{C} 6-\mathrm{C} 5$ & $122.31(11)$ & $\mathrm{C} 12-\mathrm{C} 13-\mathrm{H} 13$ & 119.46 \\
\hline $\mathrm{O} 1-\mathrm{C} 7-\mathrm{H} 7 \mathrm{a}$ & 109.47 & $\mathrm{C} 14-\mathrm{C} 13-\mathrm{H} 13$ & 119.46 \\
\hline $\mathrm{O} 1-\mathrm{C} 7-\mathrm{H} 7 \mathrm{~b}$ & 109.47 & $\mathrm{C} 9-\mathrm{C} 14-\mathrm{C} 13$ & $120.80(12)$ \\
\hline $\mathrm{O} 1-\mathrm{C} 7-\mathrm{H} 7 \mathrm{c}$ & 109.47 & C9-C14-H14 & 119.6 \\
\hline $\mathrm{H} 7 \mathrm{a}-\mathrm{C} 7-\mathrm{H} 7 \mathrm{~b}$ & 109.47 & $\mathrm{C} 13-\mathrm{C} 14-\mathrm{H} 14$ & 119.6 \\
\hline $\mathrm{H} 7 \mathrm{a}-\mathrm{C} 7-\mathrm{H} 7 \mathrm{c}$ & 109.47 & $\mathrm{O} 3-\mathrm{C} 15-\mathrm{O} 4$ & $122.60(11)$ \\
\hline $\mathrm{H} 7 \mathrm{~b}-\mathrm{C} 7-\mathrm{H} 7 \mathrm{c}$ & 109.47 & $\mathrm{O} 3-\mathrm{C} 15-\mathrm{C} 9$ & $120.04(11)$ \\
\hline $\mathrm{O} 2-\mathrm{C} 8-\mathrm{H} 8 \mathrm{a}$ & 109.47 & $\mathrm{O} 4-\mathrm{C} 15-\mathrm{C} 9$ & $117.35(11)$ \\
\hline $\mathrm{O} 2-\mathrm{C} 8-\mathrm{H} 8 \mathrm{~b}$ & 109.47 & $\mathrm{~N} 1-\mathrm{H} 4 \mathrm{~N} 1-\mathrm{H} 4 \mathrm{O} 4$ & $171(5)$ \\
\hline $\mathrm{O} 2-\mathrm{C} 8-\mathrm{H} 8 \mathrm{c}$ & 109.47 & $\mathrm{O} 4-\mathrm{H} 4 \mathrm{O} 4-\mathrm{H} 4 \mathrm{~N} 1$ & $165(5)$ \\
\hline
\end{tabular}

Hydrogen-bond geometry $\left(A,{ }^{\circ}\right)$

$\mathrm{Cg} 1$ is the centroid of the $\mathrm{C} 9-\mathrm{C} 14$ ring.

\begin{tabular}{lllll}
\hline$D-\mathrm{H} \cdots A$ & $D-\mathrm{H}$ & $\mathrm{H} \cdots A$ & $D \cdots A$ & $D-\mathrm{H} \cdots A$ \\
\hline $\mathrm{N} 1-\mathrm{H} 4 N 1 \cdots \mathrm{O} 4$ & $0.86(3)$ & $1.78(3)$ & $2.6459(14)$ & $176(2)$ \\
$\mathrm{O} 4-\mathrm{H} 4 O 4 \cdots \mathrm{N} 1$ & $0.89(3)$ & $1.77(3)$ & $2.6459(14)$ & $170(3)$ \\
$\mathrm{N} 2-\mathrm{H} 2 \mathrm{~A} \cdots \mathrm{O} 3$ & $0.92(1)$ & $1.91(1)$ & $2.8163(14)$ & $172(1)$ \\
$\mathrm{N} 2-\mathrm{H} 2 \mathrm{~B} \cdots \mathrm{O} 3^{\mathrm{i}}$ & $0.88(1)$ & $2.04(1)$ & $2.8544(14)$ & $154(1)$ \\
$\mathrm{N} 4-\mathrm{H} 4 \mathrm{~A} \cdots \mathrm{O}^{4 i}$ & $0.92(2)$ & $2.27(2)$ & $3.1625(15)$ & $164(1)$
\end{tabular}


supporting information

$\begin{array}{lllll}\mathrm{C} 7-\mathrm{H} 7 \mathrm{~B} \cdots \mathrm{O} 2^{\mathrm{iii}} & 0.96 & 2.59 & 3.4571(15) & 150 \\ \mathrm{~N} 4-\mathrm{H} 4 b \cdots C g 1^{\mathrm{iv}} & 0.89(2) & 2.724(15) & 3.5472(14) & 154.7(12)\end{array}$

Symmetry codes: (i) $-x+1,-y+2,-z$; (ii) $-x+1, y+1 / 2,-z+1 / 2$; (iii) $-x,-y+1,-z$; (iv) $-x+2, y+1 / 2,-z+1 / 2$. 